TITLE:

\title{
Phonon-phonon interactions in transition metals
}

$\operatorname{AUTHOR}(S):$

Chaput, Laurent; Togo, Atsushi; Tanaka, Isao; Hug, Gilles

\section{CITATION:}

Chaput, Laurent ... [et al]. Phonon-phonon interactions in transition metals. PHYSICAL REVIEW B 2011, 84(9): 094302.

ISSUE DATE:

2011-09

URL:

http://hdl.handle.net/2433/161782

RIGHT:

(C2011 American Physical Society 
PHYSICAL REVIEW B 84, 094302 (2011)

\title{
Phonon-phonon interactions in transition metals
}

\author{
Laurent Chaput, ${ }^{1, *}$ Atsushi Togo, ${ }^{2,3}$ Isao Tanaka, ${ }^{3,4}$ and Gilles Hug ${ }^{2}$ \\ ${ }^{1}$ Institut Jean Lamour, UMR CNRS 7198, Nancy Université, Bd. des Aiguillettes, Boîte Postale 23, \\ 54506 Vandoeuvre Les Nancy Cedex, France \\ ${ }^{2}$ Laboratoire d'Etude des Microstructures, UMR 104 ONERA-CNRS ONERA, Boîte Postale 72, 92322 Châtillon Cedex, France \\ ${ }^{3}$ Department of Materials Science and Engineering, Kyoto University, Sakyo, Kyoto 606-8501, Japan \\ ${ }^{4}$ Nanostructures Research Laboratory, Japan Fine Ceramics Center, Atsuta, Nagoya 456-8587, Japan \\ (Received 27 October 2010; revised manuscript received 20 December 2010; published 14 September 2011)
}

\begin{abstract}
In this paper the phonon self-energy produced by anharmonicity is calculated using second-order many-body perturbation theory for all bcc, fcc, and hep transition metals. The symmetry properties of the phonon interactions are used to obtain an expression for the self-energy as a sum over irreducible triplets, very similar to integration in the irreducible part of the Brillouin zone for one-particle properties. The results obtained for transition metals shows that the lifetime is on the order of $10^{-10} \mathrm{~s}$. Moreover, the Peierls approximation for the imaginary part of the self-energy is shown to be reasonable for bcc and fcc metals. For hcp metals we show that the Raman-active mode decays into a pair of acoustic phonons, their wave vector being located on a surface defined by conservation laws.
\end{abstract}

DOI: 10.1103/PhysRevB.84.094302

PACS number(s): $63.20 . \mathrm{kg}$

\section{INTRODUCTION}

Harmonic phonon calculations based on density functional theory are nowadays routinely performed for bulk solids. The dynamical matrix is either obtained from density functional perturbation theory ${ }^{1}$ or from supercell calculations. ${ }^{2,3}$ To go beyond the harmonic approximation, quasiharmonic calculations are usually performed. ${ }^{4,5}$ However, in this effective theory the phonons do not have lifetime. Ab initio anharmonic calculations, taking into account phonon-phonon interactions explicitly, are rather rare. There are noticeable exceptions with, for example, the calculations in the diamond structure of $\mathrm{Si}$ and $\mathrm{Ge},{ }^{6,7}$ and the recent study of graphite by Bonini et al. ${ }^{8}$ Such calculations give relevant information about the phonon-phonon interactions which may be hidden by the electron-phonon interaction in experiments. It is important, for example, in the understanding of energy transport in thermoelectricity. Looking at the self-energy of simple basic elements is therefore of interest.

In this paper we study the bcc, fcc, and hcp transition metals. The phonon-phonon self-energy is calculated for all metals in the crystallographic structure, stable under normal conditions. The necessary information is then extracted to obtain the decay path for a selected phonon in the Brillouin zone.

The paper is organized as follows. To explain the methodology of our calculation we first define irreducible triplets of wave vectors from the symmetry of the phonon-phonon coupling function. A formula is then obtained to calculate the $(q, \omega)$ resolved self-energy in a simple way. The results of these calculations for the transition metals are then analyzed using band decomposition and conservation surfaces for the phonons with the shorter lifetime. An approximation proposed by Peierls is also discussed. Finally, the phonon decay path generating the Raman damping is described for hcp metals.

\section{COMPUTATIONAL METHOD}

The strength of the interaction $\mathcal{F}$ between phonons of wave vectors $q, q^{\prime}, q^{\prime \prime}$ in bands $p, p^{\prime}, p^{\prime \prime}$, is given in terms of the eigenvalues $\omega_{p}(q)$ and eigenvectors $e_{p}^{\tau \alpha}(q)$ of the harmonic Hamiltonian, as well as the third derivative of the potential energy, $\Phi_{0 \tau_{1}, R_{2} \tau_{2}, R_{3} \tau_{3}}^{\alpha_{1} \alpha_{3} \alpha_{3}},{ }^{9}$

$$
\begin{aligned}
\mathcal{F}_{\left\{q q^{\prime} q^{\prime \prime}\right\}}^{\left\{p p^{\prime \prime}\right\}}= & \left(\frac{\hbar}{2}\right)^{3 / 2} \frac{1}{\sqrt{N}} \sum_{\tau_{1}, \tau_{2}, \tau_{3}} \sum_{\alpha_{1}, \alpha_{2}, \alpha_{3}} \\
& \times\left(\sum_{R_{2}, R_{3}} e^{-i q^{\prime} \cdot a_{R_{2}}-i q^{\prime \prime} \cdot a_{R_{3}}} \frac{\Phi_{0 \tau_{1}, R_{2} \tau_{2}, R_{3} \tau_{3}}^{\alpha_{1} \alpha_{2} \alpha_{3}}}{\sqrt{m_{\tau_{1}} m_{\tau_{2}} m_{\tau_{3}}}}\right) \\
& \times \frac{e_{p}^{\tau_{1} \alpha_{1}}(q) e_{p^{\prime}}^{\tau_{2} \alpha_{2}}\left(q^{\prime}\right) e_{p^{\prime \prime}}^{\tau_{3} \alpha_{3}}\left(q^{\prime \prime}\right)}{\sqrt{\omega_{p}(q) \omega_{p^{\prime}}\left(q^{\prime}\right) \omega_{p^{\prime \prime}}\left(q^{\prime \prime}\right)}} .
\end{aligned}
$$

In the above equations, $a_{i}$ are any lattice vectors of a crystal containing $N$ cells, and $\Delta r_{R \tau}^{\alpha}$ is a displacement of atom $\tau$ with mass $m_{\tau}$ in cell $R$ in the direction $\alpha$ around the equilibrium position.

According to the second-order many-body perturbation theory, the third-order Hamiltonian $\mathrm{H}_{3}$ produces the self-energy $\Sigma_{p}(q, \omega)=\Lambda_{p}(q, \omega)+i \Gamma_{p}(q, \omega)$ with the quasiparticle width decomposed as $\Gamma_{p}(q, \omega)=\sum_{p^{\prime} p^{\prime \prime}} \Gamma_{p p^{\prime} p^{\prime \prime}}(q, \omega)$ with

$$
\Gamma_{p p^{\prime} p^{\prime \prime}}(q, \omega)=\frac{\pi}{\hbar^{2}} \sum_{q^{\prime}}\left|\mathcal{F}_{\left\{-q q^{\prime} q^{\prime \prime}\right\}}^{\left\{p p^{\prime} p^{\prime \prime}\right\}}\right|^{2} f_{\left\{q q^{\prime} q^{\prime \prime}\right\}}^{\left\{p p^{\prime} p^{\prime \prime}\right\}}
$$

The function $f$ is the temperature-dependent part given in terms of the Bose-Einstein occupation factor $n_{q p}$ by

$$
\begin{aligned}
f_{\left\{q^{\prime} q^{\prime \prime}\right\}}^{\left\{p p^{\prime} p^{\prime \prime}\right\}}= & \left(n_{q^{\prime} p^{\prime}}-n_{q^{\prime \prime} p^{\prime \prime}}\right) \delta\left(\omega+\omega_{q^{\prime} p^{\prime}}-\omega_{q^{\prime \prime} p^{\prime \prime}}\right) \\
& +\frac{1}{2}\left(1+n_{q^{\prime} p^{\prime}}+n_{q^{\prime \prime} p^{\prime \prime}}\right) \delta\left(\omega-\omega_{q^{\prime} p^{\prime}}-\omega_{q^{\prime \prime} p^{\prime \prime}}\right) .
\end{aligned}
$$

However, in this paper we are only concerned with the $T=0$ limit, where the first term vanishes.

The calculation of the self-energy can be greatly improved if we use the symmetry properties of the coupling function $\mathcal{F}$. Let us denote by $\mathcal{P}$ the set of permutation operations $\mathcal{P}=$ $\left\{1, P_{23}, P_{12}, P_{13}, P_{12} P_{23}, P_{13} P_{32}\right\}$, where $P_{i j}$ switches the $i$ and $j$ element of any triplet. For example, $P_{12}\{a, b, c\}=\{b, a, c\}$. The set of rotations of the point group of the crystal is called 
$\mathcal{R}$. To make the equations more compact when such rotations are applied to a triplet of wave vectors $\left\{q q^{\prime} q^{\prime \prime}\right\}$, we will use the notation $R\left\{q q^{\prime} q^{\prime \prime}\right\}=\left\{R q R q^{\prime} R q^{\prime \prime}\right\} \quad \forall R \in \mathcal{R}$. The first vector of such a triplet is written as $R\left\{q \mid q^{\prime} q^{\prime \prime}\right\}$.

From the definition (1) of the coupling function $\mathcal{F}$, it is then straightforward to show the following properties:

$$
\begin{aligned}
& \mathcal{F}_{P\left\{q q^{\prime} q^{\prime \prime}\right\}}^{P\left\{p p^{\prime} p^{\prime \prime}\right\}}=\mathcal{F}_{\left\{q q^{\prime} q^{\prime \prime}\right\}}^{\left\{p p^{\prime \prime}\right\}} \quad \forall P \in \mathcal{P}, \\
& \mathcal{F}_{P\left\{q q^{\prime} q^{\prime \prime}\right\}}^{\left\{p p^{\prime \prime} p^{\prime \prime}\right\}}=\mathcal{F}^{P^{-1}\left\{p p^{\prime} p^{\prime \prime}\right\}} \underset{\left\{q q^{\prime} q^{\prime \prime}\right\}}{\{} \quad \forall P \in \mathcal{P} .
\end{aligned}
$$

Using the invariance of the potential energy under the spacegroup operations of the crystal and the law of transformation for the eigenvectors, ${ }^{10}$ one can also show that, $\forall R \in \mathcal{R}$,

$$
\mathcal{F}_{R\left\{q q^{\prime} q^{\prime \prime}\right\}}^{\left\{p p^{\prime}\right\}}=\mathcal{F}_{\left\{q q^{\prime} q^{\prime \prime}\right\}}^{\left\{p p^{\prime} p^{\prime \prime}\right\}} \quad \text { if } \quad q+q^{\prime}+q^{\prime \prime}=G,
$$

where $G$ is a reciprocal lattice vector.

The symmetry properties (3), (4), and (5) can now be used to define a set of irreducible triplets of wave vectors $\left\{q q^{\prime} q^{\prime \prime}\right\}$. By definition, a set of irreducible triplets is a minimal set of triplets $\left\{k k^{\prime} k^{\prime \prime}\right\}$, which sum up to a reciprocal lattice vector and can be used to generate any triplet $\left\{q q^{\prime} q^{\prime \prime}\right\}$, which also sums up to a reciprocal lattice vector, by application of the elements of $\mathcal{P} \times \mathcal{R}$. In short, $\left\{q q^{\prime} q^{\prime \prime}\right\}=R P\left\{k k^{\prime} k^{\prime \prime}\right\}$.

According to this definition it is sufficient to calculate the coupling function $\mathcal{F}$ for a set of irreducible triplets since all others can be deduced from it. If $\left\{q q^{\prime} q^{\prime \prime}\right\}=R P\left\{k k^{\prime} k^{\prime \prime}\right\}$, then one has

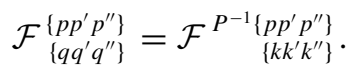

By analogy to the reduction of Brillouin zone integration to its irreducible part for properties such as the density of states, formula (2) for the self-energy is now reduced to a sum over irreducible triplets. Since $q^{\prime \prime}$ is determined from crystal momentum conservation, the summation over $q^{\prime}$ can be seen as a sum over all triplets starting with wave vector $q$. Then one can write the contribution $\Gamma_{p p^{\prime} p^{\prime \prime}}(q, \omega)$ to $\Gamma_{p}(q, \omega)$ for a phonon in band $p$ decaying to bands $p^{\prime}$ and $p^{\prime \prime}$ as

$$
\begin{aligned}
& \Gamma_{p p^{\prime} p^{\prime \prime}}(-q, \omega)=\frac{\pi}{\hbar^{2}} \sum_{\left\{k k^{\prime} k^{\prime \prime}\right\}} \sum_{P \in \mathcal{P}} \sum_{R \in \mathcal{R}}\left|\mathcal{F}_{P R\left\{k k^{\prime} k^{\prime \prime}\right\}}^{\left\{p p^{\prime} p^{\prime \prime}\right.}\right|^{2} \\
& \times f_{P R\left\{k k^{\prime} k^{\prime \prime}\right\}}^{\left\{p p^{\prime} p^{\prime \prime}\right\}} \delta_{1}\left(q, P R\left\{k \mid k^{\prime} k^{\prime \prime}\right\}\right)
\end{aligned}
$$

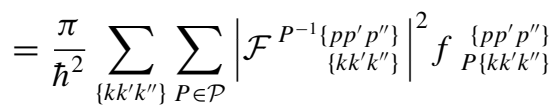

$$
\begin{aligned}
& \times \mathcal{C}_{P}\left(q,\left\{k k^{\prime} k^{\prime \prime}\right\}\right) \text {. }
\end{aligned}
$$

The $\delta_{1}$ function is different from zero if $q=P R\left\{k \mid k^{\prime} k^{\prime \prime}\right\}$ and is given by the reciprocal of the number of times the triplet $P R\left\{k k^{\prime} k^{\prime \prime}\right\}$ has been generated by application of the operations of $\mathcal{P} \times \mathcal{R}$ on $\left\{k k^{\prime} k^{\prime \prime}\right\}$. The second line is obtained using the symmetry properties (3), (4), (5), and $R P=P R$. The weight coefficients $\mathcal{C}_{P}$ are calculated once for all from $\mathcal{C}_{P}\left(q,\left\{k k^{\prime} k^{\prime \prime}\right\}\right)=\sum_{R \in \mathcal{R}} \delta_{1}\left(q, P R\left\{k \mid k^{\prime} k^{\prime \prime}\right\}\right)$, and it is also useful to note that $\mathcal{C}_{1}=\mathcal{C}_{P_{23}}, \mathcal{C}_{P_{12}}=\mathcal{C}_{P_{13} P_{32}}$, and $\mathcal{C}_{P_{13}}=\mathcal{C}_{P_{12} P_{23}}$. Equation (8) is particularly useful for computer calculations since it can easily be parallelized over irreducible triplets of wave vectors, as with single $k$ points for one-particle properties. A computer code has been implemented from these equations, and in the following it is applied to bcc, fcc, and hcp transition metals.

\section{RESULTS AND DISCUSSIONS}

With the exception of manganese, the transition metals crystallize in the bcc, fcc, and hcp structures. The secondand third-order force constants can be seen as derivatives of potential energy or derivatives of forces which are obtained from these structures using first-principle calculations. In particular, the third-order force constants are third derivatives of potential energy with respect to atomic displacements. Therefore they are calculated from forces on atoms in a supercell containing two atomic displacements. The total number of atomic displacement pairs is reduced using crystal symmetry. In our study, we employed the finite displacement method to calculate the derivatives, but to improve accuracy, displacements of plus and minus directions are applied if they are not symmetrically equivalent. The third-order force constants are usually overdetermined in this way. The tensor elements are then determined using pseudoinverse, which is the technique also employed for second-order force constants. ${ }^{2}$ The details of these calculations are given in the Appendix.

To obtain the electronic structure and forces we employed the projector augmented-wave method, ${ }^{11}$ in the framework of density functional theory, within the generalized gradient approximation of Perdew, Burke, and Ernzerhof, ${ }^{12}$ as implemented in the VASP code. ${ }^{13-15}$ Spin-polarized calculations are performed for $\mathrm{Fe}, \mathrm{Cr}, \mathrm{Ni}$, and $\mathrm{Co}$. The supercells of the bcc, fcc, and hcp structures contain 16, 32, and 16 atoms, respectively, and are limited by our computational resources. A plane-wave energy cutoff of $300 \mathrm{eV}$ is used, and $k$-point sampling meshes of $12 \times 12 \times 12,12 \times 12 \times 12$, and $16 \times 16 \times 8$ are used for bcc, fcc, and hcp supercells, respectively. The MethfesselPaxton scheme ${ }^{16}$ is employed with a smearing of $0.2 \mathrm{eV}$. The cell parameters are relaxed until the stresses become less than $10^{-3} \mathrm{GPa}$. Atomic forces are obtained with an energy convergence criterion of $10^{-8} \mathrm{eV}$, and we use displacements of $0.01 \AA$ to determine the force constants. For some metals, such as $\mathrm{Cr}$, the electronic ground state we obtain from density functional theory can be questionable. However, the forces extracted from those calculations may still be used to calculate the force constants. For example, for $\mathrm{Cr}$ we have checked that the second-order force constants give a phonon spectrum, at the point $N$ of the Brillouin zone, at most different by $6 \%$ of the experimental values. ${ }^{17}$ Since the third-order force constant is even more short ranged, we assume this approximation to be still acceptable.

According to Eq. (8) and the force constants previously calculated, we can obtain the damping functions. They are calculated for all bands and all high-symmetry points ${ }^{19}$ in the first Brillouin zone. These functions are nonzero between 0 and $2 \omega_{\max }$ but we found the center of gravity located at about $2 \bar{\omega}$, where $\bar{\omega}$ is the average phonon frequency over the Brillouin zone. Those are quite smooth functions for bcc and fcc metals, whereas they exhibit a more complicated structure for hcp metals. 


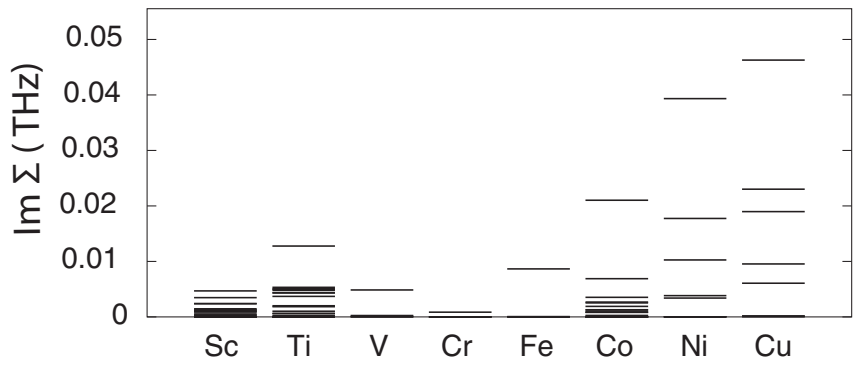

FIG. 1. Imaginary part of the self-energy in THz. The values are given for all bands and all high symmetry points of the Brillouin zones.

The probability decays of harmonic phonons are then found according to the equation $1 / 2 \tau_{q p}=\operatorname{Im} \Sigma_{q p}\left(\omega_{q p}\right)$. They are represented in Fig. 1 for all bands and all high-symmetry points of the Brillouin zones. One can see that the minimum lifetime tends to decrease toward the right end of the series. But even if most of the calculated values are greater than $0.5 \times 10^{-10} \mathrm{~s}$, one cannot distinguish a clear trend in their distribution for a given crystal symmetry.

The imaginary parts of the self-energy corresponding to the minimum lifetime are represented in Fig. 2 as functions of frequency. The vertical line shows the frequency of the irreducible representation it belongs to. They are always located within the lower tail of the self-energy.

To better understand how a harmonic phonon of frequency $\omega_{q_{0} p_{0}}$, which will be the phonon with minimum lifetime, acquires a finite lifetime $\Gamma_{q_{0} p_{0}}\left(\omega_{q_{0} p_{0}}\right)$, one should remember that this quantity is constructed from two parts. The one with the delta functions gives the decay processes which are allowed by the conservation laws, and the $\left|\mathcal{F}_{\left\{q q^{\prime} q^{\prime \prime}\right\}}^{\left\{p p^{\prime \prime}\right\}}\right|^{2}$ gives the probability for such decays to happen. The two conservation laws, for energy $\omega_{q_{0} p_{0}}=\omega_{q^{\prime} p^{\prime}}+\omega_{q^{\prime \prime} p^{\prime \prime}}$ and momentum $q_{0}=q^{\prime}+q^{\prime \prime}+G$, are coupled equations which define a conservation surface in reciprocal space: a phonon in mode $q_{0} p_{0}$ will decay in two phonons of bands $p^{\prime}$ and $p^{\prime \prime}$ with wave vectors having their extremities on that surface. For each metal, at least one couple of bands has a large probability decay. The conservation surfaces corresponding to the strongest ones are plotted in Fig. 3 and the percentages for such decays are given in the caption. In such a way one obtains a very clear view of the processes which generate the lifetime since we know the bands to which the phonons decay, as well as their wave vectors (the band indices are given in Fig. 2). For given $p^{\prime}$ and $p^{\prime \prime}$ we should, however, remember that when $p^{\prime} \neq p^{\prime \prime}$, the surface is always composed of two sheets: one centered at the origin where $q^{\prime}$ is located, and the same shifted by $q_{0}$ where $q^{\prime \prime}$ lies in. For clarity only the first one is represented in Fig. 3. All surfaces we found are open surfaces, and with the exception of Ti, they have a tube shape along $q_{0}$.

Now if we consider the frequency $\omega_{q_{0} p_{0}}$ as a variable parameter $\omega$, we generate a family of surfaces $S(\omega)$ whose shape and area give the joint density of states,

$$
D_{2}\left(q_{0}, \omega\right)=\frac{1}{\Omega} \sum_{p^{\prime} p^{\prime \prime}} \int_{S(\omega)} \frac{d S}{\nabla\left(\omega_{q^{\prime} p^{\prime}}+\omega_{q_{0}-q^{\prime} p^{\prime \prime}}\right)} .
$$

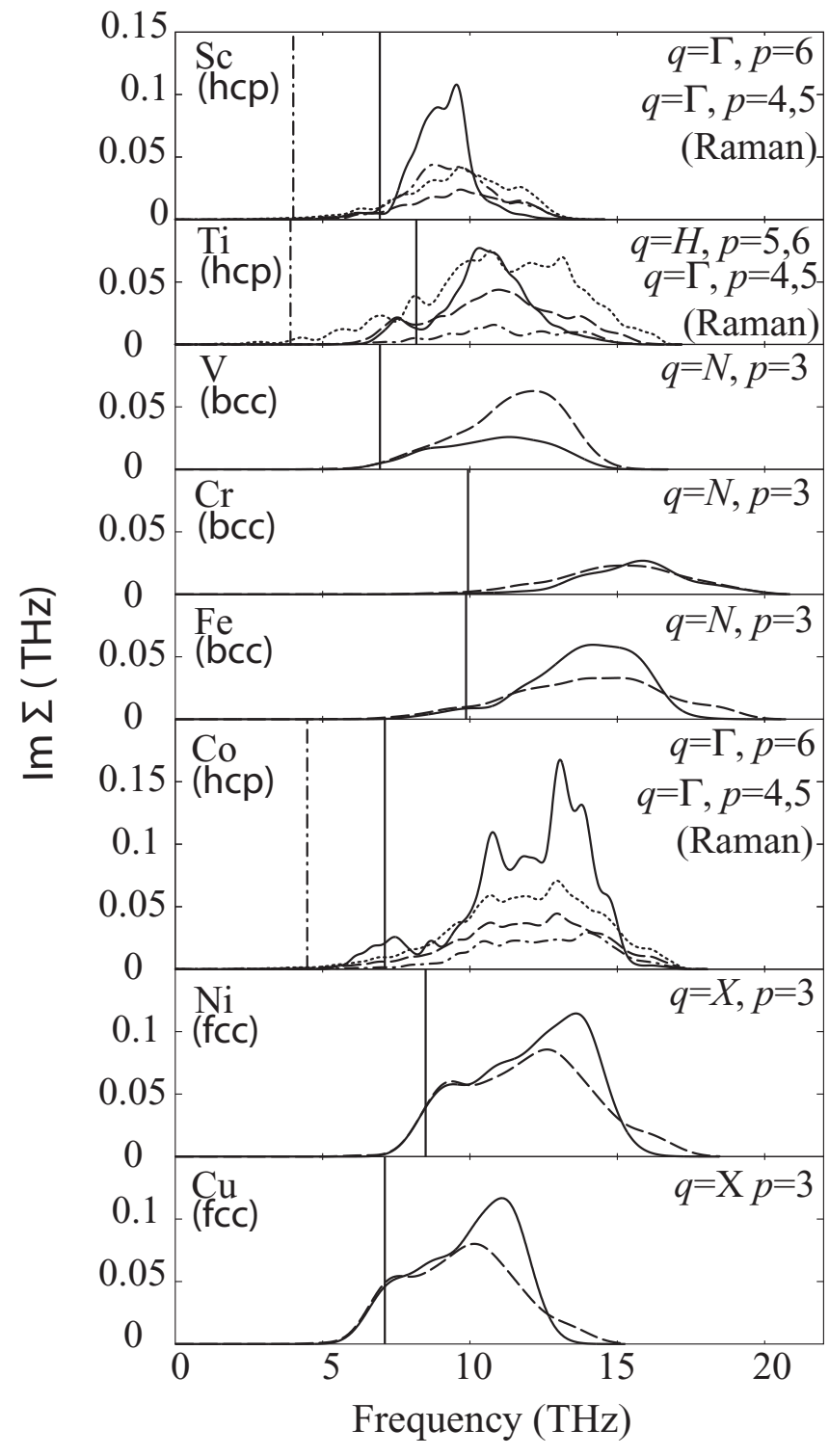

FIG. 2. The imaginary part of the self-energy calculated at the point $q$ and band $p$ is represented as a function of frequency. The continuous line is the full calculation and the dashed line corresponds to the approximation due to Peierls. The crystal structures are indicated below the chemical names. For the hcp metals the functions for the Raman-active modes are shown with a dash-dotted line and their approximation as a dotted line.

As an approximation, Peierls ${ }^{9}$ proposed the damping function $\Gamma_{q_{0} p_{0}}(\omega)$ to be proportional to the joint density of states. In fact, one can also simply fix the proportionality constant replacing $\left|\mathcal{F}_{\left\{q q^{\prime} q^{\prime \prime}\right\}}^{\left\{p p^{\prime} p^{\prime \prime}\right\}}\right|^{2}$ by its average value and rescaling by $\omega_{q p}$,

$$
\left\langle|\mathcal{F}|^{2}\right\rangle \sim \frac{\mathcal{A}}{\bar{\omega}_{q} \bar{\omega}^{2}} \rightarrow \frac{\mathcal{A}}{\omega_{q p} \bar{\omega}^{2}}
$$

where we defined $\mathcal{A}$ as $\frac{1}{\left(3 N_{a}\right)^{3}} \sum_{\tau_{1} \tau_{2} \tau_{3}} \sum_{\alpha_{1} \alpha_{2} \alpha_{3}} \sum_{R_{2} R_{3}}\left(\Phi_{0 \tau_{1}, R_{2} \tau_{2}, R_{3} \tau_{3}}^{\alpha_{1} \alpha_{2} \alpha^{2}}\right)^{2}$. It represents an averaged measure of the anharmonicity. $\bar{\omega}_{q}$ is the average value of frequencies at point $q$. 


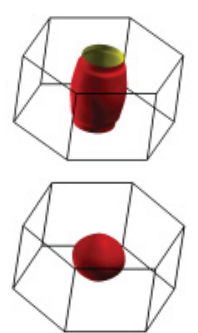

$\mathrm{Sc}$
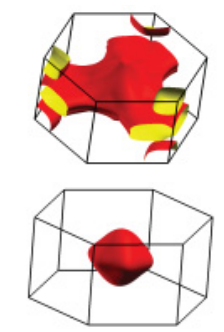

Ti
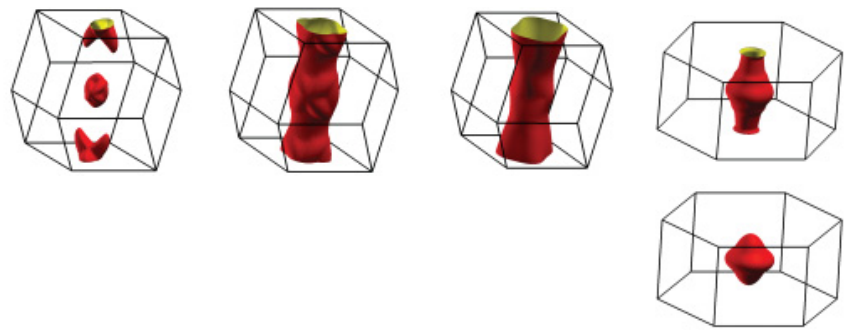

$\mathrm{Fe}$
Co
$\mathrm{Cr}$
$\mathrm{Fe}$
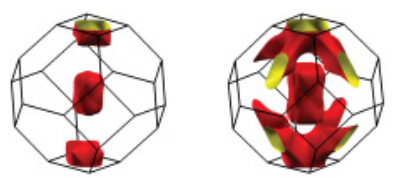

FIG. 3. (Color online) Conservation surfaces for the bands with the stronger probability decays. $q_{0}$ is chosen as the vertical direction. The surfaces are generated using the XCRYSDEN software (Ref. 18). The band indices and the contribution to the damping function (in percentages) at the harmonic frequency are as follows: $\mathrm{Sc}\left(p^{\prime}, p^{\prime \prime}=4,1 ; 36 \%\right)$, Sc-Raman $\left(p^{\prime}, p^{\prime \prime}=2,1 ; 76 \%\right)$, Ti $\left(p^{\prime}, p^{\prime \prime}=3,1 ; 27 \%\right)$, Ti-Raman $\left(p^{\prime}, p^{\prime \prime}=2,1 ; 50 \%\right), \mathrm{V}\left(p^{\prime}, p^{\prime \prime}=2,1 ; 80 \%\right), \mathrm{Cr}\left(p^{\prime}, p^{\prime \prime}=1,1 ; 50 \%\right), \mathrm{Fe}\left(p^{\prime}, p^{\prime \prime}=2,1 ; 41 \%\right), \mathrm{Co}\left(p^{\prime}, p^{\prime \prime}=4,2 ; 28 \%\right), \mathrm{Co}-\mathrm{Raman}$ $\left(p^{\prime}, p^{\prime \prime}=2,2 ; 47 \%\right)$, Ni $\left(p^{\prime}, p^{\prime \prime}=2,1 ; 59 \%\right)$, and $\mathrm{Cu}\left(p^{\prime}, p^{\prime \prime}=2,1 ; 51 \%\right)$. The three surfaces in the second row correspond to Raman decay.

The approximation is clearly good for fcc and even bcc transition metals where the anharmonicity seems to be describable by a single number $\alpha$ for these processes. However, the approximation is not accurate for hcp metals. The coupling function has a much stronger dependance on the phonon modes. This comes from the hexagonal structure which has two atoms per cell. This combination gives third-order force constants which are anisotropic, and complicated interferences between phonon eigenvectors, accoustical and optical, which cannot be removed from the calculation. We have also calculated the Raman damping functions of the hep transition metals, and the same conclusion is obtained. For this structure it is the $E_{2 g}$ mode which is Raman active. These phonons have a much longer lifetime with $2.1 \times 10^{-9} \mathrm{~s}$ for Sc, $5.8 \times 10^{-9} \mathrm{~s}$ for $\mathrm{Ti}$, and $1.8 \times 10^{-9} \mathrm{~s}$ for Co. Their conservation surfaces are presented in the second row in Fig. 3 and the damping functions are shown in Fig. 2. For this mode the stronger decay to a couple of bands $p^{\prime} p^{\prime \prime}$ is much more selective than in the case of the modes with minimum lifetime. Remarkably, an optical phonon in the Raman-active mode $E_{2 g}$ will decay into a pair of acoustic phonons in almost all cases. The wave vectors of such phonons are located on the surfaces shown in Fig. 3. These surfaces are closed, with very simple shapes. This seems to indicate that simpler models could be constructed for these decay processes.

In conclusion, we have calculated the phonon-phonon self-energy of bcc, fcc, and hcp transition metals. The decays for the phonons with minimum lifetime were studied and the conservation surfaces calculated. We found that for bcc and fcc metals the imaginary part of the self-energy is approximately proportional to the joint density of states, whereas this approximation fails in the case of hcp metals. The Raman damping was also examined for these metals and we found that a phonon decays into a pair of acoustic phonons whose wave vectors are located on spherical-like surfaces.

\section{ACKNOWLEDGMENTS}

The authors gratefully acknowledge the French Agence Nationale de la Recherche (ANR) for financial support under Contract No. 07-MAPR-0015-04, as well as the Grants-in-Aid for Scientific Research (A), Scientific Research on Priority Areas (Grant No. 474), and the Global COE Program, all from
MEXT, Japan, and the PIE - Programme Interdisciplinaire Energie of CNRS, France.

\section{APPENDIX: COMPUTATIONS OF SECOND- AND THIRD-ORDER FORCE CONSTANTS}

The potential energy of a phonon system is represented as a function of atomic positions, $V\left(\mathbf{r}_{R_{1} \tau_{1}}, \ldots, \mathbf{r}_{R_{N} \tau_{n}}\right)$, where $\mathbf{r}_{R \tau}$ is the atomic position, and $n$ and $N$ are the number of atoms in a unit cell and the number of unit cells, respectively. $\tau_{i}$ and $R_{i}$ are the indices of atoms in a unit cell and the indices of unit cells.

A force on an atom is the first derivative of the potential energy with respect to an atomic position,

$$
F_{R \tau}^{\alpha}=-\frac{\partial V}{\partial r_{R \tau}^{\alpha}}
$$

$\alpha, \beta, \ldots$, are used for the indices of Cartesian coordinates. A second-order force constant $\Phi^{\alpha \beta}$ is the second derivative of the potential energy as a function of atomic positions,

$$
\Phi_{R_{1} \tau_{1}, R_{2} \tau_{2}}^{\alpha \beta}=\frac{\partial^{2} V}{\partial r_{R_{1} \tau_{1}}^{\alpha} \partial r_{R_{2} \tau_{2}}^{\beta}}=-\frac{\partial F_{R_{1} \tau_{1}}^{\alpha}}{\partial r_{R_{2} \tau_{2}}^{\beta}},
$$

and a third-order force constant $\Phi^{\alpha \beta \gamma}$ is the third derivative of the potential energy as a function of atomic positions,

$$
\begin{aligned}
\Phi_{R_{1} \tau_{1}, R_{2} \tau_{2}, R_{3} \tau_{3}}^{\alpha \beta \gamma} & =\frac{\partial^{3} V}{\partial r_{R_{1} \tau_{1}}^{\alpha} \partial r_{R_{2} \tau_{2}}^{\beta} \partial r_{R_{3} \tau_{3}}^{\gamma}} \\
& =\frac{\partial \Phi_{R_{1} \tau_{1}, R_{2} \tau_{2}}^{\alpha \beta}}{\partial r_{R_{3} \tau_{3}}^{\gamma}} \\
& =-\frac{\partial^{2} F_{R_{1} \tau_{1}}^{\alpha}}{\partial r_{R_{2} \tau_{2}}^{\beta} \partial r_{R_{3} \tau_{3}}^{\gamma}} .
\end{aligned}
$$

Using finite differences, the derivatives in Eqs. (A2) and (A3) are approximated by

$$
\Phi_{R_{1} \tau_{1}, R_{2} \tau_{2}}^{\alpha \beta} \simeq-\frac{F_{R_{1} \tau_{1}}^{\alpha}\left[\Delta r_{R_{2} \tau_{2}}^{\beta}\right]}{\Delta r_{R_{2} \tau_{2}}^{\beta}},
$$


and

$$
\begin{aligned}
\Phi_{R_{1} \tau_{1}, R_{2} \tau_{2}, R_{3} \tau_{3}}^{\alpha \beta \gamma} & \simeq \frac{\Delta \Phi_{R_{1} \tau_{1}, R_{2} \tau_{2}}^{\alpha \beta}\left[\Delta r_{R_{3} \tau_{3}}^{\gamma}\right]}{\Delta r_{R_{3} \tau_{3}}^{\gamma}} \\
& =\frac{\Phi_{R_{1} \tau_{1}, R_{2} \tau_{2}}^{\alpha \beta}\left[\Delta r_{R_{3} \tau_{3}}^{\gamma}\right]-\Phi_{R_{1} \tau_{1}, R_{2} \tau_{2}}^{\alpha \beta}}{\Delta r^{\gamma}\left(R_{3} \tau_{3}\right)} \\
& \simeq-\frac{F_{R_{1} \tau_{1}}^{\alpha}\left[\Delta r_{R_{2} \tau_{2}}^{\beta}, \Delta r_{R_{3} \tau_{3}}^{\gamma}\right]-F_{R_{1} \tau_{1}}^{\alpha}\left[\Delta r_{R_{2} \tau_{2}}^{\beta}\right]}{\Delta r_{R_{2} \tau_{2}}^{\beta} \Delta r_{R_{3} \tau_{3}}^{\gamma}},
\end{aligned}
$$

respectively. $\Delta r^{\beta}$ and $\Delta r^{\gamma}$ correspond to the finite atomic displacements. The $\Delta r^{\beta}$ and $\Delta r^{\gamma}$, appearing in brackets, of forces and force constants, mean that the values are calculated under the displacements.

To compute the second-order force constants, we employed the technique presented by Parlinski et al. ${ }^{2}$ and the third-order force constants are obtained in a similar manner. In the following sections, the computational details are given.

\section{Computation of second-order force constants}

Second-order force constants are computed through the approximation (A4) with small displacements. For computational convenience, a second-order force constant tensor for a pair of atoms, $R_{1} \tau_{1}$ and $R_{2} \tau_{2}$, and an atomic displacement are represented by a $9 \times 1$ matrix $\mathbf{P}$ and a $3 \times 9$ matrix $\mathbf{U}$ given by

$$
\mathbf{P}\left(R_{2} \tau_{2}, R_{1} \tau_{1}\right)=\left[\Phi^{x x} \Phi^{x y} \Phi^{x z} \Phi^{y x} \Phi^{y y} \Phi^{y z} \Phi^{z x} \Phi^{z y} \Phi^{z z}\right]^{T}
$$

and

$$
\mathbf{U}\left(R_{2} \tau_{2}\right)=\left(\begin{array}{lll}
\mathbf{1} & \mathbf{0} & \mathbf{0} \\
\mathbf{0} & \mathbf{1} & \mathbf{0} \\
\mathbf{0} & \mathbf{0} & \mathbf{1}
\end{array}\right) \otimes\left[\begin{array}{lll}
\Delta r^{x} & \Delta r^{y} & \Delta r^{z}
\end{array}\right],
$$

respectively. Using these matrices, a force on an atom, which is in the form of a $1 \times 3$ matrix $\mathbf{F}$, is obtained by

$$
\mathbf{F}\left(R_{1} \tau_{1}\right)=-\mathbf{U}\left(R_{2} \tau_{2}\right) \mathbf{P}\left(R_{1} \tau_{1}, R_{2} \tau_{2}\right) .
$$

Simultaneous equations of different atomic displacements for a pair of atoms are then combined as

$$
\left(\begin{array}{c}
\mathbf{F}_{1} \\
\mathbf{F}_{2} \\
\vdots
\end{array}\right)=-\left(\begin{array}{c}
\mathbf{U}_{1} \\
\mathbf{U}_{2} \\
\vdots
\end{array}\right) \mathbf{P} .
$$

With a sufficient number of atomic displacements, Eq. (A8) may be solved by pseudoinverse such as

$$
\mathbf{P}=-\left(\begin{array}{c}
\mathbf{U}_{1} \\
\mathbf{U}_{2} \\
\vdots
\end{array}\right)^{+}\left(\begin{array}{c}
\mathbf{F}_{1} \\
\mathbf{F}_{2} \\
\vdots
\end{array}\right) .
$$

However, with the help of site-point symmetry, the required number of atomic displacements to solve the simultaneous equations may be reduced. If $R_{1}^{\prime} \tau_{1}^{\prime}$ is the image of atom $R_{1} \tau_{1}$ by a site-point symmetry operation of atom $R_{2} \tau_{2}$, Eq. (A7) becomes

$$
\begin{aligned}
\mathbf{F}\left(R_{1}^{\prime} \tau_{1}^{\prime}\right) & =-\mathbf{U}\left(R_{2} \tau_{2}\right) \mathbf{P}\left(R_{1}^{\prime} \tau_{1}^{\prime}, R_{2} \tau_{2}\right) \\
& =-\mathbf{U}\left(R_{2} \tau_{2}\right) \mathbf{A} \mathbf{P}\left(R_{1} \tau_{1}, R_{2} \tau_{2}\right),
\end{aligned}
$$

where $\mathbf{F}\left(R_{1}^{\prime} \tau_{1}^{\prime}\right)$ is the force at the atomic site obtained from the original atomic site by the site-point symmetry operation, and $\mathbf{A}$ is the $9 \times 9$ matrix that is used to rotate $\mathbf{P}$ along the site-point symmetry operation. Using Eq. (A11), the combined simultaneous equations are built such as

$$
\left(\begin{array}{c}
\mathbf{F}_{1}^{(1)} \\
\mathbf{F}_{1}^{(2)} \\
\vdots \\
\mathbf{F}_{2}^{(1)} \\
\mathbf{F}_{2}^{(2)} \\
\vdots
\end{array}\right)=-\left(\begin{array}{c}
\mathbf{U}_{1} \mathbf{A}^{(1)} \\
\mathbf{U}_{1} \mathbf{A}^{(2)} \\
\vdots \\
\mathbf{U}_{2} \mathbf{A}^{(1)} \\
\mathbf{U}_{2} \mathbf{A}^{(2)} \\
\vdots
\end{array}\right) \mathbf{P},
$$

where the superscript within parentheses gives the symmetry operation index. This is solved like Eq. (A9).

\section{Computation of third-order force constants}

The finite difference approximation for the third-order force constants is represented by matrices as

$$
\Delta \mathbf{P}\left(R_{1} \tau_{1}, R_{2} \tau_{2}\right)=\mathbf{V}\left(R_{3} \tau_{3}\right) \cdot \mathbf{Q}\left(R_{1} \tau_{1}, R_{2} \tau_{2}, R_{3} \tau_{3}\right),
$$

where $\Delta \mathbf{P}, \mathbf{V}$, and $\mathbf{Q}$ are the $9 \times 1,9 \times 27$, and $27 \times 1$ matrices corresponding to $\Delta \Phi^{\alpha \beta}, \Delta r^{\gamma}$, and $\Phi^{\alpha \beta \gamma}$, respectively, and are given by

$$
\begin{gathered}
\Delta \mathbf{P}_{\beta+3(\alpha-1)}=\Delta \Phi^{\alpha \beta}, \quad \alpha, \beta=1,2,3, \\
\mathbf{Q}_{\gamma+3(\beta-1)+9(\alpha-1)}=\Phi^{\alpha \beta \gamma}, \quad \alpha, \beta, \gamma=1,2,3,
\end{gathered}
$$

and

$$
\mathbf{V}=\left(\begin{array}{lll}
\mathbf{1} & \mathbf{0} & \mathbf{0} \\
\mathbf{0} & \mathbf{1} & \mathbf{0} \\
\mathbf{0} & \mathbf{0} & \mathbf{1}
\end{array}\right) \otimes \mathbf{U}
$$

respectively. Simultaneous equations are constructed by a similar manner to Eq. (A8) as

$$
\left(\begin{array}{c}
\Delta \mathbf{P}_{1} \\
\Delta \mathbf{P}_{2} \\
\vdots
\end{array}\right)=\left(\begin{array}{c}
\mathbf{V}_{1} \\
\mathbf{V}_{2} \\
\vdots
\end{array}\right) \mathbf{Q} .
$$

This may be solved by pseudoinverse such as

$$
\mathbf{Q}=\left(\begin{array}{c}
\mathbf{V}_{\mathbf{1}} \\
\mathbf{V}_{\mathbf{2}} \\
\vdots
\end{array}\right)^{+}\left(\begin{array}{c}
\Delta \mathbf{P}_{\mathbf{1}} \\
\Delta \mathbf{P}_{\mathbf{2}} \\
\vdots
\end{array}\right)
$$

The number of pairs of displacements to calculate can be reduced using symmetry operations that conserve a third-order force constant tensor for a triplet of atoms. If $R_{1}^{\prime} \tau_{1}^{\prime}$ is the image of atom $R_{1} \tau_{1}$ through a symmetry of the displaced structure, then one has

$$
\begin{aligned}
\Delta \mathbf{P}\left(R_{1}^{\prime} \tau_{1}^{\prime}, R_{2} \tau_{2}\right) & =\mathbf{V}\left(R_{3} \tau_{3}\right) \cdot \mathbf{Q}\left(R_{1}^{\prime} \tau_{1}^{\prime}, R_{2} \tau_{2}, R_{3} \tau_{3}\right) \\
& =\mathbf{V}\left(R_{3} \tau_{3}\right) \cdot \mathbf{B} \cdot \mathbf{Q}\left(R_{1} \tau_{1}, R_{2} \tau_{2}, R_{3} \tau_{3}\right),
\end{aligned}
$$

where $\mathbf{B}$ is the $27 \times 27$ symmetry operation matrix that transforms the tensor $\mathbf{Q}$. 
The simultaneous equations are then written in a similar manner to Eq. (A12) as

$$
\left(\begin{array}{c}
\Delta \mathbf{P}_{1}^{(1)} \\
\Delta \mathbf{P}_{1}^{(2)} \\
\vdots \\
\Delta \mathbf{P}_{2}^{(1)} \\
\Delta \mathbf{P}_{2}^{(2)} \\
\vdots
\end{array}\right)=\left(\begin{array}{c}
\mathbf{V}_{1} \mathbf{B}^{(1)} \\
\mathbf{V}_{1} \mathbf{B}^{(2)} \\
\vdots \\
\mathbf{V}_{2} \mathbf{B}^{(1)} \\
\mathbf{V}_{2} \mathbf{B}^{(2)} \\
\vdots
\end{array}\right) \mathbf{Q}
$$

and this is solved in the same way as Eq. (A18) using the pseudoinverse method.

\section{Application to silicon}

To check the validity of our approach we have computed the damping function at the $\Gamma$ for silicon, which is a wellknown case. ${ }^{7}$ The second- and third-order force constants are calculated using Eqs. (A7) and (A13). The damping function at the $\Gamma$ point is obtained from Eq. (8) and is shown in Fig. 4 as a solid line. It can be clearly seen that agreement with previous calculations $^{6,7}$ is good.

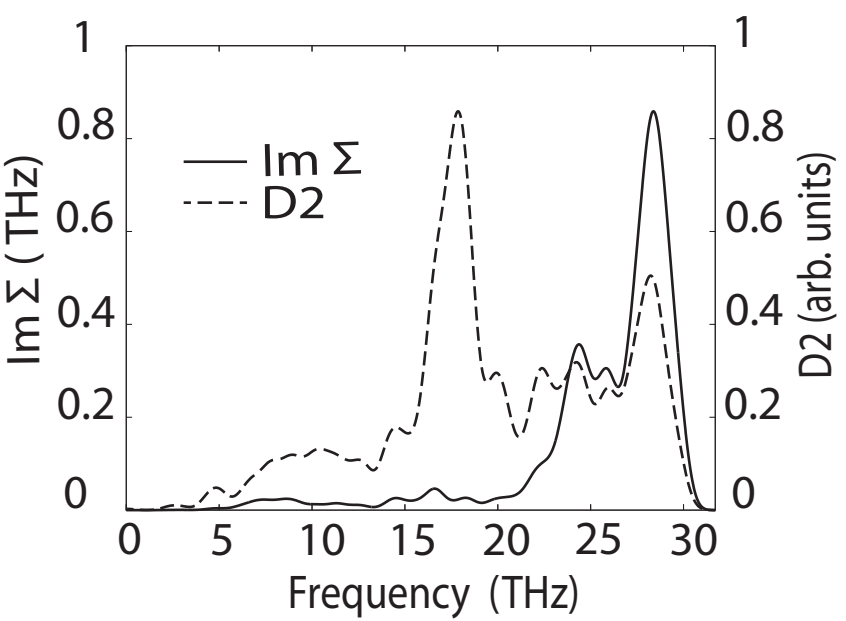

FIG. 4. The imaginary part of the self-energy $(\operatorname{Im} \Sigma)$ and the joint density of states $\left(D_{2}\right)$ at the $\Gamma$ point for silicon.

The damping function is also compared to the joint density of states (dashed line). We clearly observe an attenuation toward the low frequencies. This indicates that high-frequency phonons are more strongly damped and realistic third-order force constants are needed to achieve the description. *laurent.chaput@ijl.nancy-universite.fr

${ }^{1}$ S. Baroni, P. Giannozzi, and A. Testa, Phys. Rev. Lett. 58, 1861 (1987).

${ }^{2}$ K. Parlinski, Z.-Q. Li, and Y. Kawazoe, Phys. Rev. Lett. 78, 4063 (1997).

${ }^{3}$ A. Togo, F. Oba, and I. Tanaka, Phys. Rev. B 78, 134106 (2008).

${ }^{4}$ P. Pavone, K. Karch, O. Schutt, D. Strauch, W. Windl, P. Giannozzi, and S. Baroni, Phys. Rev. B 48, 3156 (1993).

${ }^{5}$ A. Togo, L. Chaput, I. Tanaka, and G. Hug, Phys. Rev. B 81, 174301 (2010).

${ }^{6}$ G. Deinzer, G. Birner, and D. Strauch, Phys. Rev. B 67, 144304 (2003).

${ }^{7}$ S. Narasimhan and D. Vanderbilt, Phys. Rev. B 43, 4541 (1991).

${ }^{8}$ M. Bonini, N. Lazzeri, N. Marzari, and F. Mauri, Phys. Rev. Lett. 99, 176802 (2007).
${ }^{9}$ R. E. Peierls, Quantum Theory of Solids (Oxford University Press, Oxford, 1964).

${ }^{10}$ A. A. Maradudin and S. H. Vosko, Rev. Mod. Phys. 40, 1 (1968).

${ }^{11}$ P. E. Blöchl, Phys. Rev. B 50, 17953 (1994).

${ }^{12}$ J. P. Perdew, K. Burke, and M. Ernzerhof, Phys. Rev. Lett. 77, 3865 (1996).

${ }^{13}$ G. Kresse, J. Non-Cryst. Solids 193, 222 (1995).

${ }^{14}$ G. Kresse and J. Furthmüller, Comput. Mater. Sci. 6, 15 (1996).

${ }^{15}$ G. Kresse and D. Joubert, Phys. Rev. B 59, 1758 (1999).

${ }^{16}$ M. Methfessel and A. T. Paxton, Phys. Rev. B 40, 3616 (1989).

${ }^{17}$ W. M. Shaw and L. D. Muhlestein, Phys. Rev. B 4, 969 (1971).

${ }^{18}$ A. Kokalj, Comput. Mater. Sci. 28, 155 (2003).

${ }^{19}$ Points $H, N, P$ for bcc, $K, L, W, X$ for fcc, and $\Gamma, A, H, K, M, L$ for hcp. 